\title{
Alterstice
}

Revue internationale de la recherche interculturelle

International Journal of Intercultural Research

Revista International de la Investigacion Intercultural

\section{Los intercambios en español y francés: integración lingüística e intercultural en la universidad}

\section{Jessica Payeras et Maria Elena Zapata}

Volume 8, numéro 1, 2018

Jumelage interculturel et pédagogie universitaire

URI : https://id.erudit.org/iderudit/1052607ar

DOI : https://doi.org/10.7202/1052607ar

Aller au sommaire du numéro

Éditeur(s)

Alterstice

ISSN

1923-919X (numérique)

Découvrir la revue

Citer cet article

Payeras, J. \& Zapata, M. E. (2018). Los intercambios en español y francés: integración lingüística e intercultural en la universidad. Alterstice, 8(1), 51-58. https://doi.org/10.7202/1052607ar

\section{Résumé de l'article}

El intercambio lingüístico e intercultural en la provincia de Quebec (Canadá) es un tema de gran interés ya que la recibe aproximadamente 50,000 inmigrantes anualmente, de la que 85-90 \% en Montreal. Según el censo de 2011 de Statistique Canada, los latinoamericanos representaban el 12,9 \% y este porcentaje va en aumento.

El presente artículo describe una intervención pedagógica innovadora, que se realiza en el departamento de español de la Escuela de lenguas de la Universidad de Quebec en Montreal desde el año 2007. Nos referimos a los intercambios lingüísticos e interculturales que practican los hispanohablantes inmigrantes recién llegados para establecerse en la provincia de Quebec, inscritos en un programa lingüístico del Ministerio de inmigración, diversidad e inclusión (UQAM-MIDI) y los estudiantes francófonos que estudian español como lengua extranjera en el programa de español como lengua extranjera (ELE) en la Universidad de Quebec en Montreal.

Estas actividades que se integran a los cursos universitarios de español tienen tres objetivos principales: (i) mejorar prácticas de aprendizaje accional (a través de una actividad que tiene un fin auténtico), (ii) promover el respeto mutuo de los participantes y (iii) valorar el Otro.

Los beneficios de la intervención son mutuos. Para el estudiante hispanohablante, es una manera de asegurar exitosamente el mejoramiento de su competencia lingüística en francés, su inserción académica o profesional en el nuevo contexto social donde vive. Para el estudiante francófono, estos intercambios lo llevan a entender aspectos culturales de temáticas diversas. Un objetivo adicional para los dos grupos de estudiantes es desmentir los prejuicios que hayan podido forjarse durante el aprendizaje de cada idioma, el español y el francés.
Ce document est protégé par la loi sur le droit d'auteur. L'utilisation des services d’Érudit (y compris la reproduction) est assujettie à sa politique d'utilisation que vous pouvez consulter en ligne.

https://apropos.erudit.org/fr/usagers/politique-dutilisation/ 


\section{7}

ARTICLE THÉMATIQUE

\section{Los intercambios en español y francés: integración lingüística e intercultural en la universidad*}

Jessica Payeras ${ }^{1}$ et Maria Elena Zapata ${ }^{1}$

\section{Résumé / Resumen}

El intercambio lingüístico e intercultural en la provincia de Quebec (Canadá) es un tema de gran interés ya que la recibe aproximadamente 50,000 inmigrantes anualmente, de la que 85-90 \% en Montreal. Según el censo de 2011 de Statistique Canada, los latinoamericanos representaban el 12,9 \% y este porcentaje va en aumento.

El presente artículo describe una intervención pedagógica innovadora, que se realiza en el departamento de español de la Escuela de lenguas de la Universidad de Quebec en Montreal desde el año 2007. Nos referimos a los intercambios lingüísticos e interculturales que practican los hispanohablantes inmigrantes recién llegados para establecerse en la provincia de Quebec, inscritos en un programa lingüístico del Ministerio de inmigración, diversidad e inclusión (UQAM-MIDI) y los estudiantes francófonos que estudian español como lengua extranjera en el programa de español como lengua extranjera (ELE) en la Universidad de Quebec en Montreal.

Estas actividades que se integran a los cursos universitarios de español tienen tres objetivos principales: (i) mejorar prácticas de aprendizaje accional (a través de una actividad que tiene un fin auténtico), (ii) promover el respeto mutuo de los participantes y (iii) valorar el Otro.

Los beneficios de la intervención son mutuos. Para el estudiante hispanohablante, es una manera de asegurar exitosamente el mejoramiento de su competencia lingüística en francés, su inserción académica o profesional en el nuevo contexto social donde vive. Para el estudiante francófono, estos intercambios lo llevan a entender aspectos culturales de temáticas diversas. Un objetivo adicional para los dos grupos de estudiantes es desmentir los prejuicios que hayan podido forjarse durante el aprendizaje de cada idioma, el español y el francés.

\section{Rattachement des auteures \\ ${ }^{1}$ Université du Québec à Montréal, Montréal, Canada}

\section{Correspondance}

payeras.jessica@uqam.ca

\section{Mots clés / Palabras claves}

cultura, interculturalidad, motivación, comunicación intercultural, intercambio lingüístico, diversidad, relaciones armoniosas y respeto mutuo

\section{Pour citer cet article}

Payeras, J. et Zapata, M. E. (2018). Los intercambios en español y francés: integración lingüística e intercultural en la universidad. Alterstice, 8(1), 51-58.

*Une synthèse en français est disponible dans le même numéro d'Alterstice, p. 59-62. 


\section{Introducción}

Esta intervención que se lleva a cabo en español y en francés se articula de acuerdo a las necesidades lingüísticas de sus participantes quienes desean mejorar su conocimiento lingüístico y establecer relaciones interculturales armoniosas. Además, en un futuro cercano, esta actividad se podría fácilmente traspasar a los otros programas de lengua extranjera que ofrece la Escuela de lenguas de la Universidad de Quebec en Montreal.

El marco de referencia conceptual que se utiliza en estos encuentros se basa en los siguientes conceptos: a) la cultura y la interculturalidad según la óptica de Camilleri y Cohen-Emerique (1989) y Carignan (2006); b) la comunicación intercultural y lingüística que recomienda Julien (1995a, b) y Nadon (2011) y c) la motivación intrínseca y extrínseca según el modelo de Nuttin (1991) y el enfoque de Vallerand (2006).

Desde su inserción en el programa de español lengua extranjera en el año 2007 en la Universidad de Quebec en Montreal, los intercambios lingüísticos e interculturales fueron diseñados para mejorar la competencia oral de los estudiantes y al mismo tiempo para tejer una red social exitosa entre los participantes fuera del salón de clase. La percepción de los estudiantes francófonos en cuanto al aspecto cultural del español que estaban aprendiendo en las clases era muy teórica y lejana de la realidad. Por su parte, los inmigrantes hispanohablantes que llegaban a Montreal admitían que las oportunidades para conocer residentes quebequenses eran demasiado contadas y sumamente artificiales.

Tres factores motivacionales hicieron que esta actividad tuviera un éxito casi inmediato. En primer lugar, una profesora del programa de acogida UQAM-MIDI, Diane Proulx pensaba que sus estudiantes hispanohablantes podrían beneficiarse de encuentros auténticos con estudiantes francófonos en la misma universidad donde se llevaba el programa de aprendizaje del francés como lengua extranjera del Ministerio. En segundo lugar, la profesora Jessica Payeras pensaba que sus estudiantes inscritos en los cursos de español lengua extranjera: Temas de actualidad en los países hispanohablantes y Comunicación oral y turismo podrían perfeccionar su español y, al mismo tiempo, conocer más detalles sobre las temáticas de los países latinoamericanos tratadas en el curso desde el punto de vista de sus representantes que se encontraban en situación de inmersión en Quebec. El tercer y último factor es sin ninguna duda el que tendría el impacto más fuerte: una estrecha coordinación por parte de una auxiliar de enseñanza de origen latinoamericano, María Elena Zapata. Además, en 2012, María Elena Zapata completó con éxito su informe de pasantía en el contexto de su maestría en educación en la misma universidad de Quebec en Montreal cuya problemática era el pilar de esta intervención.

Por lo tanto, todas las condiciones fueron reuniéndose en varios trimestres para llegar a la situación actual: (i) grupos de estudiantes bien motivados, (ii) una estrecha coordinación para dirigir los encuentros lingüísticos e interculturales con las bases teóricas y prácticas necesarias y (iii) el respaldo crucial de la directora del programa de español lengua extranjera en la universidad, la profesora Ayarid Guillén, cuyo curso también participó activamente en la última edición de estos encuentros.

Gracias a un acuerdo bilateral entre el programa del Ministerio UQAM-MIDI con su coordinadora anterior, la señora Marguerite Hardy y, ahora con su coordinadora actual, la señora Manon Lagarde, hemos podido compaginar necesidades y planificar estas actividades en la UQAM. Además, varios estudios sobre estas actividades han sido llevado a cabo por las autoras de este artículo. Los resultados de estos estudios han podido ser presentados en diferentes coloquios nacionales e internacionales (Payeras y Zapata 2014, 2015) y (Zapata 2008, 2016).

Las actividades que se crearon para esta intervención tienen una duración de cinco semanas consecutivas. A partir de la sexta semana de curso, en un salón de clase de la misma universidad, se identifican los horarios óptimos para que los estudiantes puedan encontrarse y trabajar en parejas. En cuanto a la logística de cada encuentro, cada uno tiene una duración de 90 minutos, divididos en dos franjas: 35 minutos dedicados a la práctica del francés y 35 minutos en la del español. La coordinadora de los encuentros se encarga de la logística completa de estos encuentros: crear listas e invitaciones personales, mandar por correo electrónico invitaciones para garantizar el 
máximo de participantes a los encuentros, llevar a cabo las actividades, motivar las presencias de los participantes y concluirlas con un informe al final de cada ciclo. En estas actividades, los estudiantes tienen la oportunidad de practicar con una persona diferente de la que han tenido en las semanas anteriores.

El artículo que presentamos está dividido en diferentes secciones. En la primera parte del artículo, 1.1, se definen los objetivos generales de la intervención y las competencias trabajadas: la oralidad, la escritura y la lectura. En la segunda parte, 1.2, se definen los principales conceptos, de cultura, interculturalidad, motivación intercultural y comunicación lingüística. En la parte, 1.3 discutimos un factor esencial: la motivación. En la cuarta parte, 1.4 compartimos una de las actividades claves: “¿Espejito, espejito, dime quién soy?". En la parte 1.5, damos a conocer los resultados de un cuestionario que completamos en el trimestre de invierno 2017 para indagar mejor la percepción de los estudiantes con respecto a esta actividad. En esta sección nos detenemos a examinar dos preguntas claves, acompañadas por el análisis de gráficos. En la sección 1.6 abordamos nuestro análisis de resultados. Finalmente, exponemos nuestras conclusiones con una reflexión que permite evaluar la importancia que posee esta intervención pedagógica en un departamento de lenguas en una universidad.

\section{Objetivos principales de la intervención y competencias trabajadas}

Los objetivos principales de esta intervención pedagógica apuntan a: 1) promover el reconocimiento mutuo y a 2) motivar a los estudiantes a compartir su cultura (diferencias y similitudes). En cada taller o encuentro, los estudiantes francófonos e hispanos descubren juntos dos culturas diversas, se reconocen y se acercan directamente a la cultura y a la lengua que estudian y perfeccionan. En cuanto a las competencias claves: la oralidad, la escritura y la lectura, ellas forman parte de la práctica lingüística destinada a mejorar la competencia general lingüística de la lengua extranjera, sea el francés o el español.

\section{Principales conceptos: cultura, interculturalidad, motivación intercultural y comunicación lingüística}

El marco conceptual de la presente intervención trabaja los conceptos de cultura e interculturalidad, motivación intrínseca y extrínseca, la comunicación intercultural y lingüística con sus tres vehículos de comunicación: la oralidad, la lectura y la escritura, que se asocian y se complementan.

Existen varias definiciones del concepto cultura de acuerdo al contexto en el que se emplee. Para esta intervención se ha elegido la siguiente: Cultura "es el conjunto más o menos ligado de significaciones adquiridas, las más persistentes y las más compartidas, que los miembros de un grupo, por su filiación a este grupo, deben propagar de manera prevalente sobre los estímulos provenientes de su medio ambiente y de ellos mismos, induciendo con respeto a estos estímulos, actitudes, representaciones y comportamientos comunes valorizados, para poder asegurar su reproducción por medios no genéticos». (Camilleri y Cohen-Emerique, 1989, p.27).

En efecto, si entendemos que la cultura es el conjunto de formas de vida, conocimientos, expresiones y sentires de un grupo social específico que tiene sus propias costumbres, creencias y hábitos, pero que comparte los valores comunes y distintos con otros grupos sociales y que se relaciona con ellos, entonces podremos afirmar que la interculturalidad, nos permite comprender mejor la relación que se establece entre dos culturas diferentes. Según Clanet (1990) el término interculturalidad, «introduce las nociones de reciprocidad en los intercambios y en la complejidad en las relaciones entre culturas [...], también se puede concebir la interculturalidad como el grupo de procesos generados por las interacciones de culturas, en un sentido de intercambios recíprocos y en una perspectiva de guardar una relativa identidad cultural compartida en relación» (Clanet, 1990, p. 21).

En la intervención pedagógica del intercambio lingüístico e intercultural, la interculturalidad no es solamente el encuentro con el otro, es también poderle decir eso que pensábamos de ese Otro. Así, compartir las similitudes y las diferencias de los dos portadores de cultura en los talleres de intercambio lingüístico e intercultural permite cumplir con los objetivos de la intervención pedagógica. Por una parte, el ambiente intercultural motiva a los estudiantes a afirmar su cultura y su identidad y a la vez establecer vínculos con otra cultura diferente (Carignan, 2006). Además, la motivación de descubrir y conocer la cultura del otro, juega un papel importante en las relaciones interculturales y armoniosas. 
Evidentemente, la comunicación intercultural y lingüística es uno de los pilares básicos en esta intervención pedagógica. "Expresarse al oral y estar en intercepción con los otros permite desarrollar competencias sociales" tal como lo explica Le Cunff y Jourdain, (1999). La práctica y el aprendizaje de una lengua en estudio permiten que la persona exprese lo que es y lo que siente.

En cada encuentro de intercambio lingüístico e intercultural se trabajan tres competencias fundamentales: la oralidad, la escritura y la lectura. Es importante destacar que en nuestros talleres hacemos más énfasis a la competencia oral en francés y en español, sin restarle importancia a la escritura y a la lectura, instrumentos primordiales en la comunicación. Al reunir estos tres elementos comunicacionales se crea lo que llamamos una "relación triangular", es decir, practicar a la vez dos lenguas como el francés y el español motivan al estudiante a querer descubrir la interculturalidad, a conocer al otro con sus valores y sus diversidades y a entender el mundo actual que busca no tener fronteras.

\section{La motivación: La llave del éxito}

En el aprendizaje de una lengua, la motivación es el motor que impulsa a una persona a elegirla, estudiarla, aprenderla y practicarla. Legendre (2005) define la motivación como "un conjunto de deseos que impulsan a una persona a realizar una tarea u objetivo para responder a una necesidad» (Legendre, 2005, p. 915). Para comprender mejor el concepto de motivación, podemos decir que este se refiere directamente al contexto del aprendizaje y de la práctica de una lengua. Vallerand (1988) plantea que existen tres tipos de motivación: la intrínseca, la extrínseca y la amotivación. Para este autor, la motivación intrínseca (MI) es el sentimiento interno que se tiene cuando se llevar a cabo una actividad por placer o por satisfacción. En otras palabras, una persona está intrínsecamente motivada "cuando ella efectúa actividades voluntarias que despiertan el interés por la actividad» (Vallerand, 1988, p.3). Vallerand (1988) define la motivación extrínseca (ME) como la motivación que reagrupa un conjunto de comportamientos que se generan por la existencia de fuerzas de control externas (Vallerand, 1988, p.7). Por su lado, la amotivación es la ausencia de toda forma de motivación intrínseca y extrínseca. En otras palabras, el individuo no percibe relación alguna entre sus acciones y los resultados obtenidos (Vallerand, 1992, p.52). Este último tipo de motivación no se ha presentado en los talleres de intercambio lingüístico e intercultural, porque tanto los estudiantes hispano hablantes como los estudiantes francófonos expresan constantemente las dos motivaciones (MI y ME).

Al analizar la motivación que poseen los hispanohablantes al aprender el francés como lengua de integración a la nueva cultura o como herramienta de comunicación en la sociedad de acogida, diremos que su motivación es extrínseca; pero a su vez, también poseen una motivación intrínseca porque les complace aprender otra lengua distinta a la materna. Si miramos el caso de los estudiantes francófonos que estudian el español como lengua extranjera, podemos decir que su motivación es intrínseca, porque sienten gusto, satisfacción y placer de conocer, hablar y practicar una lengua que los acerca al universo hispanohablante y les permite ampliar su círculo social, a su vez, sienten la motivación extrínseca cuando buscan resultados en sus estudios. Tanto los hispanohablantes como los estudiantes francófonos experimentan una motivación intrínseca cuando eligen de manera voluntaria los talleres y cuando sienten la satisfacción de progresar en el aprendizaje y en la práctica de la lengua que perfeccionan, y vivencian la motivación extrínseca cuando buscan manejar con propiedad y autonomía la lengua. Por su parte, ambos estudiantes sienten una gran pasión para compartir su cultura.

\section{Espejito, espejito, ¿Dime quién soy?}

La serie de talleres de intercambios lingüísticos e interculturales se inicia cada trimestre con una actividad que despierta una conciencia intercultural: Espejito, espejito, ¿Dime quién soy? El tema principal de esta actividad son los prejuicios y los estereotipos. Además, esta primera actividad tiene como objetivo principal que los estudiantes tengan un primer contacto entre sí, que rompan el hielo o las barreras culturales, que se conozcan y que establezcan una comunicación directa.

Desde el primer encuentro del intercambio lingüístico e intercultural, los estudiantes comparten sus experiencias de los prejuicios culturales que ellos tienen y que al expresarlos a su compañero de práctica, pueden establecer una discusión a fondo sobre el tema. 
Para llevar a cabo esta actividad pedagógica, nos basamos en los conceptos de Schneider (2005) quien define un prejuicio como un juicio a priori, una opinión preestablecida sobre un grupo de personas. Además, integramos el punto de vista de Boisaubert (2012) quien explica que cuando se habla de prejuicios se sobre entiende que se habla también de estereotipos, «que [son] un conjunto de creencias que resultan de imágenes construidas en nuestra cabeza, sin importar el grupo de personas»(p.3). El hecho de tener prejuicios sobre alguien nos lleva a crear algunos estereotipos. Bourhis (2016) por su parte opina que «los usos de estereotipos llevan a juicios erróneos, que llevan a hacer generalizaciones sobre las características de todos los individuos que son miembros de una categoría social determinada», Bourhis, 2016, p. 544.

Inspirados en el modelo de Marquis, Lavoie y Chamberland (1995), la actividad está constituida por seis etapas : 1) la distribución de un proverbio y organización de las parejas, constituidas por un hispanohablante y un estudiante francófono (5 minutos); 2) la lectura del acuerdo de participación en los talleres (5 minutos); 3) el desarrollo de la actividad en francés (35 minutos) y en español (35 minutos); 4) las respuestas a las preguntas y a los comentarios (5 minutos); 5) la conclusión de la actividad (5 minutos); y 6) la explicación de las consignas de la tarea o deber que el participante debe entregar en el siguiente taller y conclusión del taller ( 5 minutos).

\section{Cuestionario}

Al finalizar cada serie de intercambios lingüísticos e interculturales español-francés, se les pide formalmente a los participantes completar un formulario de evaluación de las diferentes actividades. Como los formularios son anónimos, se establece un grado de confianza en las respuestas sabiendo que la identidad de los participantes nunca está comprometida y se utilizan simplemente con el objetivo de mejorar la calidad de los encuentros. Compartimos aquí dos de los puntos más destacados.

\section{Apreciación de los intercambios lingüísticos e interculturales}

Para esta pregunta, que indaga sobre la apreciación de los intercambios, la respuesta que arroja el cuestionario es $96 \%$ afirmativa como lo muestra el gráfico 1.1. Desde su implantación, observamos que los estudiantes hacen todo lo posible para venir a estos encuentros, incluyendo la difícil modificación de sus horarios y actividades familiares. Aunque pareciera que esto fuera una dificultad considerable, al final de cada ciclo de actividades, nos piden de organizar más intercambios. Obviamente, la dificultad más grande de parte de la logística administrativa es coordinar que los temas, los intereses y los horarios de las actividades se inserten bien en los objetivos de los programas tanto del Ministerio como de los cursos de español lengua extranjera (ELE) en la UQAM, donde el programa nació.

Gráfico 1. Representación de la apreciación de los intercambios lingüísticos e interculturales

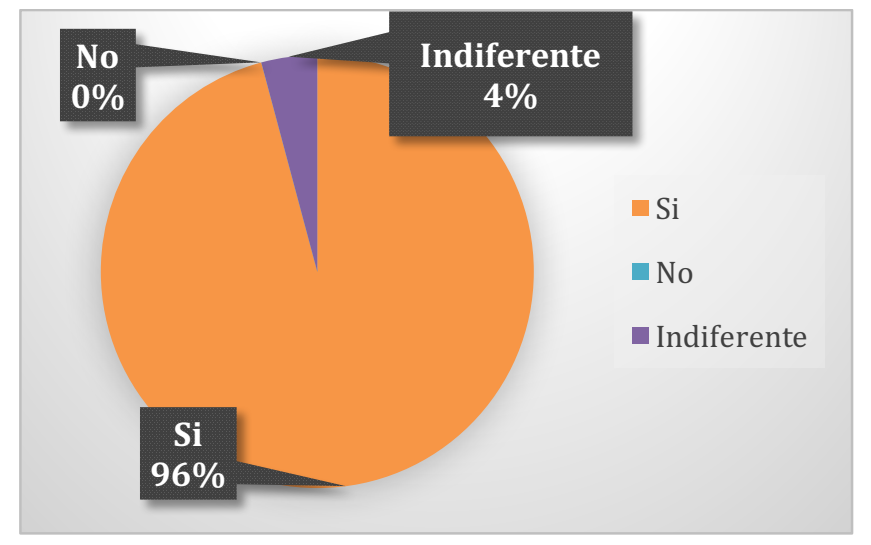

\section{Grupo Facebook}

En este punto, queremos mencionar una iniciativa que marcó un nuevo paso en la cohesión y camaradería entre los participantes de estas actividades. En la tercera semana de los encuentros una estudiante del programa de español lengua extranjera creó y lideró un grupo Facebook que sirviera de red social entre los participantes de los 
intercambios. En los mensajes se veía una apreciación y una colaboración entre estudiantes hispanohablantes y los estudiantes francófonos. En este grupo se seguía de manera espontánea la práctica lingüística que había comenzado en las clases. También se compartieron fotos, videos de canciones populares y proverbios, pertenecientes a las dos culturas, lo que causó mucho entusiasmo y, consecuentemente, mucha participación interactiva. Una verdadera red social empezó a gestarse. Pasemos a la siguiente sección que presenta nuestro análisis, conclusiones y reflexiones finales.

\section{Análisis de resultados}

Si hacemos un análisis de las experiencias de los encuentros que hemos llevado a cabo, podemos asegurar que los profesores de los estudiantes que participan en estos encuentros deben cumplir los objetivos lingüísticos y culturales de esta actividad. De punto de vista formativo, existe una rúbrica dedicada a la participación en estos encuentros que se traduce en un $5 \%$ de la nota final en el plan de curso en el cual la actividad se inserta. Este $5 \%$ incluye la corrección y entrega de informes. Después de cada encuentro, cada participante redacta un informe que lo hace poner en práctica los conocimientos lingüísticos que se han trabajado en dicho taller. Estos informes son corregidos en dos versiones: la primera donde simplemente se señalan los errores gramaticales y la segunda que es la versión definitiva para que los estudiantes hagan un esfuerzo suplementario de auto-corrección. Estas evaluaciones concretas conjunto con los cuestionarios cualitativos dan una idea real de la comprensión de los temas tratados.

De punto de vista administrativo, la dirección del programa de español está presente desde la planificación hasta los aspectos de coordinación de horarios y participantes. Es importante subrayar que los estudiantes han sido seleccionados en base al nivel de competencia lingüística en el idioma que están aprendiendo para poder interactuar fácilmente al oral: intermedio-intermedio o avanzado-avanzado. Las profesoras de ambos programas examinan el expediente de los cursos seguidos por cada participante para garantizar un nivel óptimo.

Algunas dificultades iniciales tuvieron que ser superadas en la oferta de estas actividades. Al principio, el programa tenía el objetivo simplemente de hacer la práctica oral de las estructuras lingüísticas y de llenar tal vez objetivos de socialización muy generales. Los encuentros en los primeros años dependían de la disponibilidad de los participantes. Como consecuencia, en varias ocasiones, teníamos encuentros desequilibrados donde a una persona hispana se le asignaban a dos estudiantes francófonos porque teníamos un número desigual de participantes. Esta situación encontró su solución poniendo parámetros estrictos en el proceso de selección y de control del nivel lingüístico de los participantes. Por otro lado, varios encuentros con la dirección del programa del programa UQAM-MIDI eran necesarios para explicar el proyecto, ya que el personal cambiaba con frecuencia.

En cuanto a los estudiantes francófonos de esta experiencia, una de las observaciones más interesantes es que ellos pasan de la etapa de estudiante 'pasivo' de una lengua extranjera a un actor inteligente y conocedor de hablantes nativos de español y de su idiosincrasia. Por el lado de los estudiantes hispanohablantes, estos asumen un nuevo rol de actores principales insertados en su nuevo medio ambiente y conocen a las personas quebequenses que tejen esta sociedad y logran adoptar un rol significativo en ella, respetando sus valores y su cultura.

Podemos constatar que los intercambios lingüísticos e interculturales descritos en este artículo, se imbrican en el marco teórico de Lussier (2015), que propone que la competencia de comunicación intercultural se manifiesta en tres planos diferentes pero complementarios: (i) conocimiento cultural, (ii) adaptación de comportamientos culturales y (iii) actuaciones culturales en sociedad.

En el caso de nuestras intervenciones, los estudiantes tienen la oportunidad de reflexionar sobre estos tres planos en sus encuentros. Durante estas actividades ambos participantes se cuestionan sobre la identidad cultural del Otro; aprenden los patrones de comportamientos culturales en ambos contextos $y$, finalmente, comprenden suficientemente las diferencias culturales para saber interactuar en sociedad. 


\section{Conclusiones}

Podemos concluir que los objetivos lingüísticos y culturales que respaldan estas actividades se han cumplido en los diferentes trimestres. Sin duda alguna, podemos constatar que estos encuentros lingüísticos e interculturales contribuyen a un mejor aprendizaje y práctica del español como lengua extranjera y del francés como lengua segunda o tercera. Si hablamos de motivación, podemos asegurar que los participantes tanto hispanohablantes como francófonos salen cambiados de estos encuentros. Muchos de ellos despejan los prejuicios estereotipados que poseían antes de conocer al Otro y participan en un proceso de auto-reflexión sobre sus propios valores. Para los hispanohablantes en particular, esta experiencia les permite salir de su zona 'protegida' del salón de clase y aventurarse en un mundo real. A ambos grupos les da oportunidad de mejorar sus conocimientos lingüísticos, pero más allá de abrir sus mentes y horizontes y, tal vez, lo más importante, encuentran su lugar en la sociedad.

En cuanto pistas para un desarrollo en el futuro, pensamos que este enfoque se puede fácilmente extender a los otros idiomas enseñados en la universidad. Últimamente se están dando intercambios entre estudiantes de portugués y francés, inglés y francés y, en algunos trimestres, francés y español con el programa de francés lengua extranjera. Sin embargo, el placer por estas actividades y su apreciación a nivel académico, profesoral y ministerial no deja de sorprender y de crear nuevas expectativas.

\section{Références bibliographiques / Bibliografía}

Boisaubert, L. (2012). Publicación en línea : Dossier pédagogique : Stéréotypes et préjugés. https://www.mahj.org/sites/mahj.org/files/atoms/files/stereotypes_prejuges.pdf

Bourhis, R. et Gagnon, A. (2006). Les préjugés, la discrimination et les relations intergroupes. Dans Les fondements de la psychologie sociale ( $2^{\mathrm{e}}$ éd., chapitre 13, p. 534-548). Montréal : Gaëtan Morin /Chenelière Éducation,.

Camilleri, C. et Cohen-Emerique, M. (1989). Chocs de cultures: Concepts et enjeux pratiques de l'interculturel. Paris: L'Harmattan.

Carignan, N. (2006). Est-ce possible d'apprendre à vivre ensemble? Un projet stimulant pour les futurs enseignants et les nouveaux arrivants. Actes du colloque, quelle immigration, pour quel Québec? dans le cadre du $25^{\mathrm{e}}$ anniversaire de la table de concertation des réfugiés et des immigrants (TCRI), 23-24 mars 2005. Montréal.

Carignan, N., Guillot, M.C. et Deraîche, M. (2015). Jumelages interculturels : communication, inclusion et intégration. Québec : Presses de l'Université du Québec.

Chaves, R-M., Favier, L. et Pélissier, S. (2012). L'interculturel en classe. Grenoble : Presses universitaires de Grenoble.

Clanet, C. (1990). L'interculturel. Introduction aux approches interculturelles en éducation et en sciences humaines. Toulouse : Presses universitaires du Mirail.

Gaudet, E. (2015). Relations interculturelles : comprendre pour mieux agir (3e éd.). Québec : Thomson/Groupe Modulo.

Johnston, P. H. et Nadon, Y. (2010). L'importance des mots. Le langage au cœur de la relation élève-enseignant. Montréal : Chenelière Éducation.

Le Cunff, C. et Jourdain, P. (1999). Enseigner l'oral à l'école primaire. Paris: Hachette Éducation.

Lussier, D. (2015). Les compétences culturelles, interculturelles et transculturelles [Internet]. Association canadienne d'éducation de langues française, Série de documents « Comprendre la construction identitaire ». www.acelf.ca/outils/comprendre.

Marquis, D., Lavoie, L. et Chamberland, G. (1995). 20 Formules pédagogiques. Montréal : Presses de l'Université du Quebec à Montréal.

Montgomery, C. et Bourassa-Dansereau, C. (dir.) (2017). Mobilités internationales et intervention interculturelle : Théories, expériences et pratiques. Québec : Presses de l'Université du Québec. 
Payeras, J. et Zapata, M. E. (2014). «El intercambio lingüístico e intercultural». Presentación en el coloquio de I'ASELE, Universidad Carlos III, Madrid, Espagne.

Payeras, J. et Zapata, M. E. (2015). « Los intercambios lingüísticos : una propuesta para trabajar el español y la interculturalidad en clase ». Presentación en el 4to Encuentro Internacional de Español como Lengua Extranjera “Enseñanza, Aprendizaje y Evaluación”, Universidad Sergio Arboleda, Bogotá, Colombia.

Vallerand, R. J. (dir.) (2006). Les fondements de la psychologie sociale. Montréal : Gaëtan Morin.

Zapata, M. E. et Carignan, N. (2012). Les jumelages linguistiques: une expérience d'interculturalité à Montréal. Multiculturalisme, interculturalisme et la compréhension interculturelle entre les communautés et les intervenants. Canadian Diversity/ Diversité canadienne, 9(2).

Zapata, M. E. (2016). L'harmonisation des relations interculturelles à travers les jumelages linguistiques et interculturels. Presentación en el $84^{\mathrm{e}}$ congrés de l'ACFAS, "Points de rencontres » UQAM, Montréal, Canada. 